\title{
SISTEMA RADICULAR DE PLANTAS DE COBERTURA SOB COMPACTAÇÃO DO SOLO
}

\author{
WAINER G. GONÇALVES ${ }^{1}$, RODRIGO L. JIMENEZ ${ }^{1}$, JERÔNIMO V. DE ARAÚJO \\ FILHO $^{1}$, RENATO L. DE ASSIS ${ }^{2}$, GILSON P. SILVA ${ }^{3}$, FÁBIO R. PIRES ${ }^{3}$
}

RESUMO: Com o objetivo de avaliar a capacidade de crescimento de raízes em camadas de solo compactadas, quatro espécies de plantas de cobertura (amaranto, milheto ADR 500, capim pé-degalinha e kenaf) foram cultivadas em anéis de PVC, com níveis de compactação em subsuperfície (densidade do solo: 1,$18 ; 1,34 ; 1,51$ e $1,60 \mathrm{Mg} \mathrm{m}^{-3}$ ), sendo o experimento conduzido em casa de vegetação, utilizando-se de um Latossolo Vermelho distroférrico. A camada compactada em subsuperfície foi restritiva ao crescimento de raízes das espécies estudadas, ocasionando a concentração de raízes na camada superficial. O milheto ADR 500 e o amaranto foram as espécies que se destacaram na produção de massa seca da parte aérea e conseguiram desenvolver-se nas camadas compactadas e abaixo delas. O milheto ADR500 apresentou maior densidade de comprimento radicular em todas as camadas. O capim pé-de-galinha e o amaranto tiveram comportamento semelhante quanto à densidade de comprimento radicular. O capim pé-de-galinha e o kenaf apresentaram menor massa seca de raízes em relação às demais espécies. O kenaf apresentou menores valores de massa seca da parte aérea, mas não foi afetado pela presença de camadas compactadas.

PALAVRAS-CHAVE: resistência do solo, densidade de comprimento radicular, crescimento radicular.

\section{ROOT SYSTEM OF COVER CROPS UNDER SOIL COMPACTION}

ABSTRACT: With the objective of evaluate the root growth capacity in the compacted soil layer, four vegetal species of the cover crops (amaranth, pearl millet ADR500, finger millet and kenaf) were cultivated in columns of PVC with increasing levels of subsurface compaction (soil bulk densities: $1.18 ; 1.34 ; 1.51$ and $1.60 \mathrm{Mg} \mathrm{m}^{-3}$ ). The experiment was carried out in a greenhouse conditions, using a Dusky Red Latosol. The subsurface compacted layer was restrictive to the roots growth of the studied species, causing the root concentrating to the surface. Pearl millet ADR500 and the amaranth were the species that had detached in the production of dry matter weight and developed itself in the compacted layers and below of them. Pearl of millet ADR500 presented the biggest root length density in all layers. Finger millet and the amaranth had similar behavior in relation to the shoot length root. Finger millet and kenaf had presented minor dry matter weight of root in relation to the other species. The kenaf presented minors values of dry matter, but it was not affected by the presence of compacted layers.

KEYWORDS: soil resistance, root length density, growth root.

\section{INTRODUÇÃO}

A compactação do solo assume crescente importância em face à mecanização nas atividades agrícolas, plantio direto e aumento na intensidade de cultivo (safra e safrinha) acarretando elevação na resistência do solo ao crescimento de raízes, fazendo com que as mesmas se desenvolvam mais

\footnotetext{
${ }^{1}$ Estudante de graduação do Curso de Agronomia da Universidade de Rio Verde, Rio Verde - GO.

${ }^{2}$ Eng ${ }^{\mathrm{o}}$ Agrícola, Professor do Departamento de Agronomia, Universidade de Rio Verde, Rio Verde - GO, assis@ fesurv.br.

${ }^{3}$ Eng $^{\mathrm{O}}$ Agrônomo, Professor do Departamento de Agronomia, Universidade de Rio Verde, Rio Verde - GO, gilson@fesurv.br, frpires@fesurv.br.

Recebido pelo Conselho Editorial em: 28-2-2005

Aprovado pelo Conselho Editorial em: 3-2-2006
} 
superficialmente; além disso, a compactação reduz a infiltração da água no solo e aumenta o risco de erosão e de déficits hídrico e nutricional nas plantas (ROSOLEM et al., 1994; MORAES et al., 1995).

A formação de camadas compactadas superficial e subsuperficialmente, limitação comumente observada em solos de cerrado intensivamente cultivados, tem sido a principal restrição ao aprofundamento radicular e considerada como causa primária da erosão (CASSEL, 1980; KEMPER \& DERPSCH, 1981 e CINTRA \& MIELNICZUK, 1983).

O solo compactado apresenta redução no número de macroporos e elevação na sua densidade. Como resultado dessas alterações, as raízes sofrem modificações morfológicas e fisiológicas, por vezes específicas a cada espécie ou cultivar, a fim de se adaptarem (MÜLLER et al., 2001).

Para evitar a compactação ou para minimizá-la, a diminuição das operações agrícolas, por si só, não é condição suficiente. São necessárias rotações de culturas envolvendo espécies que produzam grande quantidade de massa para a cobertura do solo e que, principalmente, possuam sistema radicular profundo e com grande volume, capaz de promover canais, diminuindo, assim, a compactação (CARDOSO et al., 2003). O aporte de resíduos culturais das plantas de cobertura, incluindo o seu sistema radicular, constitui-se numa alternativa eficiente e de baixo custo de adicionar compostos orgânicos ao solo, resultando em melhor estruturação do solo.

Dessa forma, ao contrário do que ocorre com o uso de subsoladores, as plantas descompactadoras podem proporcionar rompimento mais uniforme da camada compactada, além de contribuírem para a melhoria do estado de agregação do solo (CAMARGO \& ALLEONI, 1997). Quando as raízes que penetraram em camadas compactadas se decompõem, elas deixam canais que podem melhorar o movimento da água e a difusão dos gases no perfil do solo, além de servirem de caminhos para a penetração radicular de culturas subseqüentes (BARLEY, 1954).

A utilização de espécies de cobertura capazes de romper camadas compactadas vem assumindo papel importante, principalmente na região de cerrado, devido ao aumento de áreas sob plantio direto; entretanto, pouco se conhece sobre a capacidade de crescimento de sistema radicular da maioria dessas espécies em camadas compactadas de solo. Todavia, não se deve restringir às espécies tradicionais, uma vez que é possível a adoção de várias outras alternativas de seqüência e épocas de semeadura, as quais resultam em diversidade de opções e diminuição de riscos (SALTON, 1999). Existem espécies alternativas (amaranto, kenaf, capim pé-de-galinha, entre outras) pouco conhecidas na agricultura brasileira, mas que apresentam importância como fonte de alimento em outras partes do mundo (SPEHAR et al., 1997; SPEHAR \& CABEZAS, 1999), e que são adaptadas às condições edafoclimáticas do cerrado, principalmente pela elevada produção de fitomassa e tolerância ao déficit hídrico.

O presente estudo visou a identificar espécies de plantas de cobertura com potencial para romper camadas de solo compactadas em subsuperfície, por meio da avaliação do crescimento radicular.

\section{MATERIAL E MÉTODOS}

O experimento foi realizado no período de dezembro de 2003 a janeiro de 2004, em casa de vegetação, na Faculdade de Agronomia da Universidade de Rio Verde - GO. O delineamento experimental foi em blocos casualizados, em esquema fatorial $4 \times 4$ (quatro espécies de cobertura e quatro níveis de densidade do solo), em subsuperfície, com quatro repetições. As densidades do solo (Ds) foram: 1,$18 ; 1,34 ; 1,51$ e $1,60 \mathrm{Mg} \mathrm{m}^{-3}$, representando o grau de compactação de $70 \% ; 80 \% ; 90 \%$ e $95 \%$, respectivamente. O grau de compactação do solo, que consiste numa relação entre a densidade do solo atual e a densidade do solo máxima extraída da curva de compactação (VARGAS, 1977), foi obtido da fórmula: $\mathrm{GC}=\left(\mathrm{Ds} / \mathrm{Ds}_{\text {máx }}\right) 100$, em que, GC é o grau de compactação; Ds é a densidade do solo $\left(\mathrm{Mg} \mathrm{m}^{-3}\right)$, e $\mathrm{Ds}_{\text {máx }}$ é a densidade do solo máxima $\left(\mathrm{Mg} \mathrm{m}^{-3}\right)$. 
Foram utilizadas quatro espécies de plantas de cobertura: amaranto (Amaranthus cruentus L.), capim pé-de-galinha (Eleusine coracana), milheto (Pennisetum americanum (L.) Leek var. ADR 500) e kenaf (Hybiscus cannabinus).

Utilizaram-se amostras deformadas de solo provenientes do horizonte A $(0-0,20 \mathrm{~m})$ de um Latossolo Vermelho distroférrico textura muito argilosa. Após secagem ao ar, o solo foi passado em peneira com $2 \mathrm{~mm}$ de malha e submetido à caracterização química e textural (Tabela 1), conforme metodologia proposta pela EMBRAPA (1997).

A saturação por bases do solo foi elevada para 50\%, conforme método apresentado em RAIJ \& QUAGGIO (1983), por meio da adição de calcário. O solo foi umedecido a $80 \%$ da capacidade de campo e armazenado em sacos plásticos para incubação úmida por 15 dias. O teor de água na capacidade de campo foi determinado segundo método da EMBRAPA (1997).

TABELA 1. Caracterização química e análise textural do Latossolo Vermelho distroférrico.

\begin{tabular}{|c|c|c|c|c|c|c|c|c|c|c|c|c|c|}
\hline $\mathrm{pH}$ & $\mathrm{P}$ & $\mathrm{H}+\mathrm{Al}$ & $\mathrm{Al}$ & $\mathrm{K}$ & $\mathrm{Ca}$ & $\mathrm{Mg}$ & CTC & $\mathrm{V}$ & M.O. & Areia & Silte & Argila & C.C. ${ }^{*}$ \\
\hline$\left(\mathrm{CaCl}_{2}\right)$ & $\mathrm{mg} \mathrm{dm}^{-3}$ & & & $\mathrm{cmol}_{\mathrm{c}} \mathrm{C}$ & $m^{-3}---$ & & & $\%$ & $\mathrm{~g} \mathrm{dm}^{-3}$ & -------י- & $\mathrm{g} \mathrm{kg}^{-1}$ & & $\mathrm{~g} \mathrm{~kg}^{-1}$ \\
\hline 4,2 & 1,2 & 3,5 & 0,2 & 0,02 & 0,20 & 0,10 & 3,83 & 8,56 & 18,0 & 190 & 180 & 630 & 290 \\
\hline
\end{tabular}

* Teor de água na capacidade de campo a $-0,01 \mathrm{MPa}$.

Os recipientes utilizados constaram da sobreposição de três anéis de PVC de $0,10 \mathrm{~m}$ de diâmetro interno, tendo o superior e o inferior $0,15 \mathrm{~m}$ de altura cada um, os quais receberam solo com densidade de $1,1 \mathrm{Mg} \mathrm{m}^{-3}$ (Figura 1). $\mathrm{O}$ anel intermediário, com 0,035 $\mathrm{m}$ de altura, recebeu os tratamentos com densidades entre 1,18 e $1,60 \mathrm{Mg} \mathrm{m}^{-3}$.

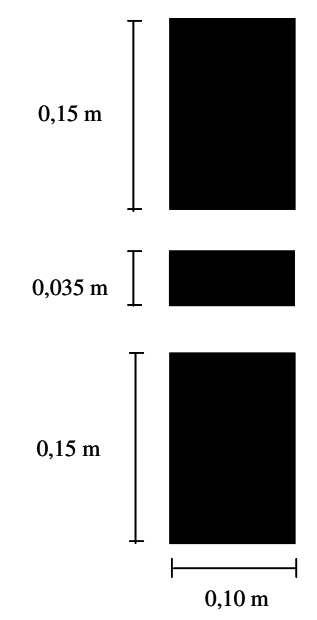

FIGURA 1. Ilustração representativa da montagem dos anéis.

Para a obtenção dos níveis de compactação desejados, foram adicionadas quantidades calculadas de solo ao anel central. Para isso, inicialmente, foi determinada a curva de compactação do solo pelo método de Proctor normal (NOGUEIRA, 1995), que consiste em compactar amostra de solo dentro de um cilindro, com volume aproximado de $1,0 \mathrm{dm}^{3}$, em três camadas sucessivas, com a aplicação de 25 golpes com um soquete com peso de $2,5 \mathrm{~kg}$ à altura de queda de $0,30 \mathrm{~m}$. O ensaio foi repetido para diferentes umidades (cinco corpos-de-prova com umidades crescentes), obtendo-se, assim, para cada valor de umidade à base de peso $(\mathrm{U})$, uma densidade do solo. Os resultados de umidade à base de peso versus densidade do solo foram ajustados a um polinômio de $2^{\mathrm{o}}$ grau $\left(\mathrm{Ds}=\mathrm{aU}^{2}+\mathrm{bU}+\mathrm{c}\right)$, utilizando o "software" Excell, obtendo-se a curva de compactação, a umidade ótima $\left(U_{\text {ót }}\right)$ e a densidade do solo máxima $\left(D s_{\text {máx }}\right)$ pelas expressões: $U_{\text {ót }}=-b / 2 a$ e $D_{\text {máx }}=-\left(b^{2}-4 a c\right) / 4 a$, segundo IEZZI (1994). O valor 
da $\mathrm{Ds}_{\text {máx }}$ determinada em laboratório foi de $1,68 \mathrm{Mg} \mathrm{m}^{-3}$ (média de três repetições). Procedeu-se à compactação do anel intermediário por meio de golpes sucessivos de uma massa de ferro até atingir a espessura de 3,5 cm, com a quantidade de solo para obter as densidades finais desejadas: 1,18; 1,34; 1,51 e $1,60 \mathrm{Mg} \mathrm{m}^{-3}$, estando o solo com umidade de $75 \%$ da capacidade de campo. Em seguida, foi medida a resistência do solo à penetração com um penetrômetro de bolso, marca Humboldt MFG.Co., modelo H-4200, com dez repetições, no momento da montagem do experimento (Tabela 2).

TABELA 2. Resistência do solo à penetração, considerando as diferentes densidades na camada compactada e a umidade à base de peso de $21,75 \mathrm{~g} \mathrm{~kg}^{-1}$.

\begin{tabular}{cc}
\hline Densidade do Solo & Resistência do Solo à Penetração \\
\hline $\mathrm{Mg} \mathrm{m}^{-3}$ & $\mathrm{MPa}$ \\
\hline 1,18 & 0,12 \\
1,34 & 0,23 \\
1,51 & 0,37 \\
1,60 & 0,45 \\
\hline
\end{tabular}

Para evitar o crescimento radicular na interface solo-PVC do anel compactado, foi utilizado o caulim que, umedecido, formou uma pasta plástica aderida à parede interna do tubo de PVC. A montagem das colunas foi realizada com o auxílio de fita adesiva para unir os anéis.

O plantio foi realizado em 19 de dezembro, sendo conduzidas duas plantas por coluna de solo. Foi realizada a adubação com $150 \mathrm{mg} \mathrm{dm}^{-3}$ de $\mathrm{N}$ (uréia), $150 \mathrm{mg} \mathrm{dm}^{-3}$ de $\mathrm{P}$ (superfosfato simples) e $150 \mathrm{mg} \mathrm{dm}^{-3}$ de $\mathrm{K}$ (cloreto de potássio).

Decorrido o período de 30 dias, realizou-se a coleta da parte aérea das plantas de cobertura, seccionando-as rente ao solo. O material vegetal foi seco em estufa $\left(65^{\circ} \mathrm{C}\right.$ por $\left.72 \mathrm{~h}\right)$, pesado e quantificada a massa de matéria seca da parte aérea.

$\mathrm{O}$ sistema radicular foi dividido em três partes, correspondendo às camadas superior, inferior e intermediária (compactada), e separada do solo por lavagem. Após secagem em estufa, determinaramse a produção de massa seca das raízes (MSR) e o comprimento radicular com a utilização do programa Quant Root v.1.0 (AMARAL, 2002). Foi calculada, em seguida, a densidade de comprimento radicular por meio da divisão do comprimento radicular encontrado em cada camada pelo volume do respectivo anel de PVC, ou seja, $1.178 \mathrm{~cm}^{3}$, para as camadas superior e inferior, e $275 \mathrm{~cm}^{3}$, para a camada compactada. Os resultados foram submetidos à análise de variância e as médias comparadas pelo teste Tukey, a 5\% de probabilidade, sendo os níveis de compactação submetidos à análise de regressão.

\section{RESULTADOS E DISCUSSÃO}

Ocorreram interações significativas entre as espécies e a densidade do solo para a variável massa seca de raízes na camada compactada e na camada inferior, e para densidade do comprimento radicular em todas as camadas. Em relação à produção de matéria seca da parte aérea, houve diferença apenas entre as espécies (Tabela 3).

Observou-se que, com o aumento da densidade na camada compactada, ocorreu aumento de massa seca de raízes das plantas (MSR) na camada superior dos vasos (Tabela 4), indicando maior concentração de raízes no anel superior, o que ocorreu em todas as espécies e em praticamente todas as densidades. Resultados semelhantes foram observados por MÜLLER et al. (2001), utilizando adubos verdes de inverno, e MORAES et al. (1995) com a cultura da soja. No presente experimento, esses resultados foram devidos à maior resistência à penetração de raízes na $\mathrm{Ds}=1,60 \mathrm{Mg} \mathrm{m}^{-3}$. As espécies 
que apresentaram maior acúmulo de MSR na camada compactada foram o amaranto e o milheto ADR 500.

TABELA 3. Significância da análise de variância de massa seca de raízes, matéria seca da parte aérea e densidade de comprimento radicular nas diferentes camadas.

\begin{tabular}{|c|c|c|c|c|}
\hline \multirow{2}{*}{ Camada } & \multicolumn{3}{|c|}{ Causas de Variação } & \multirow[t]{2}{*}{ C.V. (\%) } \\
\hline & Espécies (E) & Densidade (D) & $E \times D$ & \\
\hline \multicolumn{5}{|c|}{ Massa seca da parte aérea } \\
\hline & $* *$ & ns & ns & 14,28 \\
\hline \multicolumn{5}{|c|}{ Massa seca de raízes } \\
\hline Camada superior & $* *$ & $* *$ & ns & 18,67 \\
\hline Camada compactada & $*$ & ns & $* *$ & 33,52 \\
\hline Camada inferior & $* *$ & $* *$ & * & 34,47 \\
\hline \multicolumn{5}{|c|}{ Densidade do comprimento radicular } \\
\hline Camada superior & $* *$ & $*$ & $* *$ & 24,14 \\
\hline Camada compactada & $* *$ & $* *$ & $* *$ & 18,43 \\
\hline Camada inferior & $* *$ & ** & ** & 20,30 \\
\hline
\end{tabular}

${ }^{\mathrm{ns}}$ não significativo; * $\mathrm{e}^{* *}$ significativo a $5 \%$ e $1 \%$, respectivamente, pelo teste $\mathrm{F}$.

O amaranto, apesar do maior acúmulo de MSR na camada superior (Tabela 4), apresentou, abaixo da camada compactada, menores acúmulos de MSR do que o milheto ADR 500, para os menores níveis de compactação.

O capim pé-de-galinha e o kenaf não foram sensíveis ao aumento da compactação do solo, apesar de apresentarem menor acúmulo de MSR em todas as camadas, não devendo ser indicados para a utilização em sistemas de rotação de culturas com a finalidade de descompactação do solo em razão de deixarem, no solo, menores extensões de poros após a decomposição de raízes.

O milheto ADR 500 destacou-se entre as espécies estudadas quanto ao acúmulo de MSR, na camada compactada e na inferior, no maior nível de compactação (Tabela 4). Esses resultados evidenciam o potencial do milheto ADR 500 para crescimento em solos compactados. As espécies utilizadas apresentaram resposta diferenciada em relação à massa seca da parte aérea (MSPA) (Tabela 4). O amaranto e o milheto ADR 500 foram as espécies que se destacaram na produção de MSPA. O capim pé-de-galinha apresentou valores intermediários de produção de MSPA, bem como na produção de matéria seca de raízes. O kenaf foi a espécie que apresentou menor acúmulo de MSPA para todas as densidades e com pequena variação na MSPA com o aumento da densidade do solo na camada compactada.

É possível notar, ainda, que a densidade de $1,34 \mathrm{Mg} \mathrm{m}^{-3}$ resultou em maior acúmulo de MSPA que as demais densidades para o capim pé-de-galinha, o que está de acordo com as observações de MÜLLER et al. (2001), de que o nível intermediário de densidade do solo pode promover melhor contato solo-raiz, culminando com melhores condições de absorção de água e nutrientes, não prejudicando drasticamente o crescimento das raízes. 
TABELA 4. Massa seca de raízes (MSR) das plantas nas camadas superior, compactada e inferior, e massa seca da parte aérea (MSPA) das plantas de cobertura considerando as densidades do solo na camada compactada ${ }^{(1)}$.

\begin{tabular}{|c|c|c|c|c|}
\hline \multirow{2}{*}{ Espécie } & \multicolumn{3}{|c|}{ Camada } & \multirow[b]{2}{*}{ MSPA } \\
\hline & Superior & Compactada & Inferior & \\
\hline & \multirow{2}{*}{\multicolumn{4}{|c|}{$\begin{array}{c}\text { MSR } \\
\text { Densidade do solo }\left(1,18 \mathrm{Mg} \mathrm{m}^{-3}\right)\end{array}$}} \\
\hline & & & & \\
\hline Amaranto & $2,41 \mathrm{a}$ & $0,28 \mathrm{a}$ & $0,82 b$ & $15,35 \mathrm{a}$ \\
\hline Milheto ADR 500 & $1,44 b$ & $0,22 \mathrm{ab}$ & $1,32 \mathrm{a}$ & $14,23 \mathrm{a}$ \\
\hline Capim Pé-de-Galinha & $0,82 \mathrm{c}$ & $0,12 b c$ & $0,44 b c$ & $7,23 b$ \\
\hline \multirow[t]{2}{*}{ Kenaf } & $0,59 \mathrm{c}$ & $0,06 \mathrm{c}$ & $0,21 \mathrm{c}$ & $4,53 \mathrm{c}$ \\
\hline & \multicolumn{4}{|c|}{ Densidade do solo $\left(1,34 \mathrm{Mg} \mathrm{m}^{-3}\right)$} \\
\hline Amaranto & $2,04 \mathrm{a}$ & $0,26 \mathrm{a}$ & $0,90 \mathrm{~b}$ & $13,48 \mathrm{a}$ \\
\hline Milheto ADR 500 & $1,41 b$ & $0,19 \mathrm{ab}$ & $1,67 \mathrm{a}$ & $12,78 \mathrm{a}$ \\
\hline Capim Pé-de-Galinha & $0,92 \mathrm{c}$ & $0,12 b c$ & $0,45 c$ & $7,70 \mathrm{~b}$ \\
\hline Kenaf & $0,62 \mathrm{c}$ & $0,07 \mathrm{c}$ & $0,19 \mathrm{c}$ & $4,35 \mathrm{c}$ \\
\hline & \multicolumn{4}{|c|}{ Densidade do solo $\left(1,51 \mathrm{Mg} \mathrm{m}^{-3}\right)$} \\
\hline Amaranto & $2,35 \mathrm{a}$ & $0,15 \mathrm{a}$ & $0,70 \mathrm{ab}$ & $13,28 \mathrm{a}$ \\
\hline Milheto ADR 500 & $1,56 b$ & $0,20 \mathrm{a}$ & $0,80 \mathrm{a}$ & $12,75 \mathrm{a}$ \\
\hline Capim Pé-de-Galinha & $0,85 \mathrm{c}$ & $0,15 \mathrm{a}$ & $0,35 b c$ & $6,90 \mathrm{~b}$ \\
\hline Kenaf & $0,68 \mathrm{c}$ & $0,05 \mathrm{~b}$ & $0,07 \mathrm{c}$ & $3,65 \mathrm{c}$ \\
\hline & \multicolumn{4}{|c|}{ Densidade do solo $\left(1,60 \mathrm{Mg} \mathrm{m}^{-3}\right)$} \\
\hline Amaranto & $2,70 \mathrm{a}$ & $0,17 \mathrm{~b}$ & $0,89 \mathrm{a}$ & $12,63 \mathrm{a}$ \\
\hline Milheto ADR 500 & $1,81 b$ & $0,30^{\mathrm{a}}$ & $1,07 \mathrm{a}$ & $14,40 \mathrm{a}$ \\
\hline Capim Pé-de-Galinha & $1,01 \mathrm{c}$ & $0,08 \mathrm{bc}$ & $0,30 \mathrm{~b}$ & $7,08 \mathrm{~b}$ \\
\hline Kenaf & $0,90 \mathrm{c}$ & $0,04 \mathrm{c}$ & $0,09 b$ & $5,13 b$ \\
\hline
\end{tabular}

Os resultados da densidade de comprimento radicular (DCR) seguiram a mesma tendência do acúmulo de massa de matéria seca de raízes para as espécies estudadas, com exceção do amaranto (Tabela 5).

O milheto ADR 500 apresentou, independentemente do valor da densidade do solo na camada compactada, maiores valores de DCR em relação às outras camadas e às outras espécies. $\mathrm{O}$ milheto ADR 500 apresentou, na camada compactada, diminuição da DCR com o aumento da densidade do solo e apresentou, no maior nível de compactação $\left(\mathrm{Ds}=1,60 \mathrm{Mg} \mathrm{m}^{-3}\right.$ ), maior DCR na camada inferior que as demais espécies.

A densidade de comprimento radicular é uma informação relevante que reflete a capacidade de determinada espécie emitir raízes e ocupar/explorar o solo, o que resulta em benefícios à planta e ao solo. Pode-se inferir, portanto, que o milheto ADR 500 deixaria no solo, nessas condições, maiores extensões de poros após o processo de decomposição. Esses poros são importantes para a infiltração de água e difusão de gases, concorrendo para a melhoria das condições físicas do solo e para melhor crescimento do sistema radicular das culturas em seqüência, o que está de acordo com observações de campo de PASQUALETTO et al. (1999) e PASQUALETTO \& COSTA (2001), em que o milheto demonstrou ser a espécie com maior potencial para o manejo de solos com compactação em subsuperfície. 
TABELA 5. Distribuição da densidade de comprimento radicular das plantas de cobertura nas camadas superior, compactada e inferior, levando em consideração as densidades do solo na camada compactada ${ }^{(1)}$.

\begin{tabular}{|c|c|c|c|}
\hline \multirow{2}{*}{ Espécie } & \multicolumn{3}{|c|}{ Camada } \\
\hline & Superior & Compactada & Inferior \\
\hline & \multirow{2}{*}{\multicolumn{3}{|c|}{$\begin{array}{c}\mathrm{cm} \mathrm{cm}^{-3} \\
\text { Densidade do solo }\left(1,18 \mathrm{Mg} \mathrm{m}^{-3}\right)\end{array}$}} \\
\hline & & & \\
\hline Amaranto & $1,45 \mathrm{a}$ & $1,26 \mathrm{~b}$ & $0,89 b$ \\
\hline Milheto ADR 500 & $1,72 \mathrm{a}$ & $4,40 \mathrm{a}$ & $3,25 \mathrm{a}$ \\
\hline Capim Pé-de-Galinha & $1,11 \mathrm{ab}$ & $0,95 b c$ & $1,07 \mathrm{~b}$ \\
\hline \multirow[t]{2}{*}{ Kenaf } & $0,68 \mathrm{~b}$ & $0,56 \mathrm{c}$ & $0,36 \mathrm{c}$ \\
\hline & \multicolumn{3}{|c|}{ Densidade do solo $\left(1,34 \mathrm{Mg} \mathrm{m}^{-3}\right)$} \\
\hline Amaranto & $1,48 b$ & $1,84 \mathrm{~b}$ & $0,70 \mathrm{bc}$ \\
\hline Milheto ADR 500 & $2,16 \mathrm{a}$ & $3,82 \mathrm{a}$ & $3,10 \mathrm{a}$ \\
\hline Capim Pé-de-Galinha & $1,19 b$ & $1,29 b c$ & $1,10 b$ \\
\hline \multirow[t]{2}{*}{ Kenaf } & $0,83 b$ & $0,80 \mathrm{c}$ & $0,38 \mathrm{c}$ \\
\hline & \multicolumn{3}{|c|}{ Densidade do solo $\left(1,51 \mathrm{Mg} \mathrm{m}^{-3}\right)$} \\
\hline Amaranto & $2,12 \mathrm{a}$ & $1,18 \mathrm{~b}$ & $0,65 c$ \\
\hline Milheto ADR 500 & $2,30 \mathrm{a}$ & $2,80 \mathrm{a}$ & $1,81 \mathrm{a}$ \\
\hline Capim Pé-de-Galinha & $1,03 \mathrm{~b}$ & $1,47 b$ & $1,14 b$ \\
\hline \multirow[t]{2}{*}{ Kenaf } & $0,96 \mathrm{~b}$ & $0,57 \mathrm{c}$ & $0,32 \mathrm{c}$ \\
\hline & \multicolumn{3}{|c|}{ Densidade do solo $\left(1,60 \mathrm{Mg} \mathrm{m}^{-3}\right)$} \\
\hline Amaranto & $1,14 b$ & $1,03 b$ & $0,85 b$ \\
\hline Milheto ADR 500 & $3,07 \mathrm{a}$ & $2,53 \mathrm{a}$ & $2,52 \mathrm{a}$ \\
\hline Capim Pé-de-Galinha & $1,22 \mathrm{~b}$ & $0,94 b$ & $0,83 b$ \\
\hline Kenaf & $1,04 \mathrm{~b}$ & $0,71 b$ & $0,24 c$ \\
\hline
\end{tabular}

O amaranto apresentou, na camada compactada, diminuição da DCR nos maiores níveis de compactação do solo (Ds $=1,51$ e $1,60 \mathrm{Mg} \mathrm{m}^{-3}$ ), devido ao fato de essa espécie possuir raiz principal pivotante e menor quantidade de raízes de menor diâmetro comparada ao milheto.

O kenaf apresentou sistema radicular pouco desenvolvido, o que ocasionou valores de DCR significativamente menores que os das demais espécies. No maior nível de compactação $\left(\mathrm{Ds}=1,60 \mathrm{Mg} \mathrm{m}^{-3}\right)$, o kenaf apresentou redução significativa na DCR na camada inferior, o que demonstra a sua suscetibilidade de crescimento em ambiente compactado.

O capim pé-de-galinha apresentou valores intermediários de DCR, uma vez que, apesar de ser uma gramínea, apresentando maior número de raízes por volume de solo, mostrou-se medianamente sensível à compactação para valores de DCR.

Os diferentes níveis de compactação na camada compactada proporcionaram reduções significativas na densidade de comprimento radicular para o milheto ADR 500 e aumento para o kenaf na camada superior (Figura 2). 


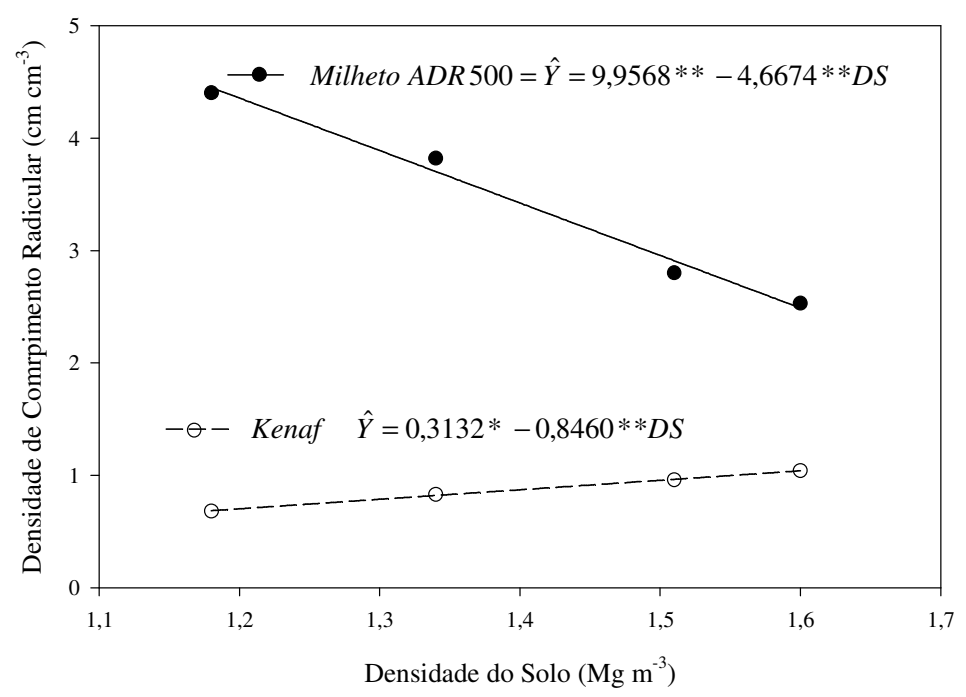

FIGURA 2. Equações de regressão da densidade de comprimento radicular para o milheto ADR 500, na camada compactada, e para kenaf, na camada superior, em função da densidade do solo. $* *$ e $*$ significativo a 1 e $5 \%$, respectivamente.

\section{CONCLUSÕES}

A camada compactada em subsuperfície foi restritiva ao crescimento de raízes das espécies estudadas, ocasionando a concentração de raízes próxima à superfície.

O milheto ADR 500 e o amaranto foram as espécies que se destacaram na produção de massa de matéria seca da parte aérea e conseguiram desenvolver-se nas camadas compactadas e abaixo delas. $\mathrm{O}$ milheto ADR 500 apresentou, nas camadas superior, compactada e inferior, maior densidade de comprimento radicular.

O capim pé-de-galinha e o amaranto tiveram comportamentos semelhantes quanto à densidade de comprimento radicular.

O capim pé-de-galinha e o kenaf apresentaram menor massa seca de raízes em relação às demais espécies. Quanto à massa seca da parte aérea, o kenaf apresentou menores valores, mas não foi afetado pela presença de camadas compactadas.

\section{REFERÊNCIAS}

AMARAL, J.F.T. Eficiência de produção de raízes, absorção, translocação e utilização de nutrientes em cultivares de café arábica. 2002. 97 f. Tese (Doutorado em Solos e Nutrição de Plantas) Universidade Federal de Viçosa, Viçosa, 2002.

BARLEY, K.P. Effect of root growth and decay on the permeability of a synthetic sandy loam. Soil Science, Baltimore, v.78, n.2, p.205-10, 1954.

CAMARGO, O.A.; ALLEONI, L.R.F. Compactação do solo e o desenvolvimento das plantas. Piracicaba: ESALQ, 1997. 132 p.

CARDOSO, E.G.; ZOTARELLI, L.; PICCININ, J.; TORRES, J. Distribuição do sistema radicular da cultura da soja em função do manejo do solo. In: CONGRESSO BRASILEIRO DE CIÊNCIA DO SOLO, 29., 2003, Ribeirão Preto. Anais... Ribeirão Preto: Sociedade Brasileira de Ciência do Solo, 2003. 1 CD-ROM. 
CASSEL, D.K. Effects of plowing depth and deep incorporation of lime and phosphorus apon physical and chemical properties of two Coastal Plain Soil after 15 years. Soil Science Society of American Journal, Madison, v.44, n.1, p.89-95, 1980.

CINTRA, F.L.D.; MIELNICZUKL, J. Potencial de algumas espécies vegetais para recuperação de solos com propriedades físicas degradadas. Revista Brasileira de Ciência do Solo, Campinas, v.7, p.197-201, 1983.

EMPRESA BRASILEIRA DE PESQUISA AGROPECUÁRIA - EMBRAPA. Centro Nacional de Pesquisa de Solos. Manual de métodos de análise de solo. 2. ed. Rio de Janeiro, 1997. 212 p.

IEZZI, G. Fundamentos de matemática Elementar 6 - complexos, polinômios e equações. 6 . ed. São Paulo: Atual, 1994. 242 p.

KEMPER, B.; DERPSCH, R. Soil compaction and root growth in Paraná. In: RUSSEL, R.S. (Ed.) The soil/root system in relation to Brazilian agriculture. Londrina: Fundação Instituto Agronômico do Paraná, 1981. p.81-101.

MORAES, M.H.; BENEZ, S.H.; LIBARDI, P.L. Efeitos da compactação em algumas propriedades físicas do solo e seu reflexo no desenvolvimento das raízes de plantas de soja. Bragantia, Campinas, v.54, n.2, p.393-403, 1995.

MÜLLER, M.M.L.; CECCON, G.; ROSOLEM, C.A. Influência da compactação do solo em subsuperfície sobre o crescimento aéreo e radicular de plantas de adubação verde de inverno. Revista Brasileira de Ciência do Solo, Viçosa, v.25, n.3, p.531-8, 2001.

NOGUEIRA, J.B. Mecânica dos solos: ensaios de laboratório. São Carlos: Escola de Engenharia de São Carlos, USP, 1995. 248 p.

PASQUALETTO, A.; COSTA, L.M. da. Contribuição de safrinhas em sucessão à cultura da soja em plantio direto sobre a resistência à penetração do solo. Pesquisa Agropecuária Tropical, Goiânia, v.31, n.1, p.55-9, 2001.

PASQUALETTO, A.; COSTA, L.M. da.; SILVA, A.A. da; SEDIYAMA, C.S. Influência de culturas de safrinhas em sucessão à cultura do milho (Zea mays L.) no sistema plantio direto sobre a resistência à penetração do solo. Pesquisa Agropecuária Tropical, Goiânia, v.29, n.2, p.27-31, 1999.

RAIJ, B. Van; QUAGGIO, J.A. Métodos de análise de solo para fins de fertilidade. Boletim Técnico Instituto Agronômico, Campinas, v.81, n.8, p.1-31, 1983.

ROSOLEM, C.A.; VALE, L.S.R.; GRASSI FILHO, H.; MORAES, M.H. Sistema radicular e nutrição do milho em função da calagem e da compactação do solo. Revista Brasileira de Ciência do Solo, Campinas, v.18, p.491-7, 1994.

SALTON, J.C. Opções de safrinha para agregação de renda nos cerrados. In: ENCONTRO REGIONAL DE PLANTIO DIRETO NO CERRADO, PLANTIO DIRETO NA INTEGRAÇÃO LAVOURA-PECUÁRIA, 1999, Uberlândia. Anais... Uberlândia : Universidade Federal de Uberlândia, 1999. p.189-200.

SPEHAR, C.R.; CABEZAS, W.A.R.L. Introdução e seleção de espécies para a diversificação do sistema produtivo nos cerrados. In: ENCONTRO REGIONAL DE PLANTIO DIRETO NO CERRADO, PLANTIO DIRETO NA INTEGRAÇÃO LAVOURA-PECUÁRIA, 1999, Uberlândia. Anais... Uberlândia: Universidade Federal de Uberlândia, 1999. p.179-88.

SPEHAR, C.R.; SANTOS, R.L.B.; SOUZA, P.I.M. Novas espécies de plantas de cobertura para plantio direto. In: SEMINÁRIO INTERNACIONAL DO SISTEMA DE PLANTIO DIRETO, 2., 1997, Brasília. Anais... Passo Fundo: Embrapa Trigo, 1997. p.169-72.

VARGAS, M. Introdução à mecânica dos solos. São Paulo: McGraw-Hill do Brasil, 1977. 509 p. 